\title{
A structural model of PpoA derived from SAXS-analysis-Implications for substrate conversion
}

\author{
Christian Koch ${ }^{\mathrm{a}, 1}$, Giancarlo Tria ${ }^{\mathrm{b}, \mathrm{f}, 1}$, Alistair J. Fielding ${ }^{\mathrm{c}, 2}$, Florian Brodhun ${ }^{\mathrm{a}}$, Oliver Valerius ${ }^{\mathrm{d}}$, \\ Kirstin Feussner a , Gerhard H. Braus ${ }^{\mathrm{d}}$, Dmitri I. Svergun ${ }^{\mathrm{b}, *}$, Marina Bennati ${ }^{\text {cee }}$, Ivo Feussner ${ }^{\text {a, } * *}$ \\ a Georg-August-University, Albrecht-von-Haller-Institute, Department of Plant Biochemistry, Justus-von-Liebig-Weg 11, D-37077 Goettingen, Germany \\ ${ }^{b}$ EMBL Hamburg, BioSAXS group, Notkestrasse 85, D-22603 Hamburg, Germany \\ c Max-Plank-Institute for Biophysical Chemistry, Research group Electron Paramagnetic Resonance, Am Fassberg 11, D-37077 Goettingen, Germany \\ d Georg-August-University, Institute for Microbiology and Genetics, Griesebachstr. 8, D-37077 Goettingen, Germany \\ e Georg-August-University, Institute for Organic and Biomolecular Chemistry, Tammannstrasse 2, D-37077 Göttingen, Germany \\ ${ }^{\mathrm{f}}$ University of Hamburg, Centre for Bioinformatics, Bundesstrasse 43, D-20146, Hamburg, Germany
}

\section{A R T I C L E I N F O}

\section{Article history:}

Received 4 February 2013

Received in revised form 29 May 2013

Accepted 7 June 2013

Available online 21 June 2013

\section{Keywords:}

Cytochrome P450

Dioxygenase

Double electron-electron resonance

Oxylipin

Psi-factor producing oxygenase

Small-angle X-ray scattering

\begin{abstract}
A B S T R A C T
In plants and mammals, oxylipins may be synthesized via multi step processes that consist of dioxygenation and isomerization of the intermediately formed hydroperoxy fatty acid. These processes are typically catalyzed by two distinct enzyme classes: dioxygenases and cytochrome P450 enzymes. In ascomycetes biosynthesis of oxylipins may proceed by a similar two-step pathway. An important difference, however, is that both enzymatic activities may be combined in a single bifunctional enzyme. These types of enzymes are named Psi-factor producing oxygenases (Ppo). Here, the spatial organization of the two domains of PpoA from Aspergillus nidulans was analyzed by small-angle X-ray scattering and the obtained data show that the enzyme exhibits a relatively flat trimeric shape. Atomic structures of the single domains were obtained by template-based structure prediction and docked into the enzyme envelope of the low resolution structure obtained by SAXS. EPR-based distance measurements between the tyrosyl radicals formed in the activated dioxygenase domain of the enzyme supported the trimeric structure obtained from SAXS and the previous assignment of Tyr374 as radical-site in PpoA. Furthermore, two phenylalanine residues in the cytochrome P450 domain were shown to modulate the specificity of hydroperoxy fatty acid rearrangement.
\end{abstract}

(C) 2013 Elsevier B.V. All rights reserved.

\section{Introduction}

Products of lipid peroxidation are commonly termed oxylipins [1] and may possess pivotal functions as signal molecules [2-4]. Biosynthesis of these compounds is often a sequential process involving a

Abbreviations: AOS, allene oxide synthase; DEER, double electron-electron resonance; DES, divinyl ether synthase; DiHODE, dihydroxy linoleic acid; DiHOME, dihydroxy oleic acid; $D_{\max }$, maximal particle dimension; DOX, dioxygenase; EPR, electron paramagnetic resonance; $\mathrm{Et}_{2} \mathrm{O}$, diethyl ether; HPL, hydroperoxide lyase; $\mathrm{H}(\mathrm{P}) \mathrm{ODE}$, hydro(pero)xy linoleic acid; KODE, oxo-linoleic acid; KOME, oxo-oleic acid; LOX, lipoxygenase; MALDI-TOF, matrix-assisted laser desorption/ionization-time of flight; MW, molecular weight; PGIS, prostacyclin synthase; psi, precocious sexual inducer; Ppo, psi-factor producing oxygenase; PGHS, prostaglandin endoperoxide synthase; $\mathrm{R}_{\mathrm{g}}$, radius of gyration; SAXS, small-angle X-ray scattering; TXAS, thromboxane synthase; $V_{p}$, particle volume

* Corresponding author. Tel.: +49 4089902 125; fax: +49 4089902149

** Corresponding author. Tel.: + 4955139 5743; fax: +49551 395749

E-mail addresses: Svergun@EMBL-Hamburg.De (D.I. Svergun), ifeussn@uni-goettingen.de (I. Feussner).

${ }^{1}$ These authors contributed equally to the article.

2 Present address: University of Manchester, Photon Science Institute, Oxford Road, M13 9PL Manchester, UK. co-factor (heme or non-heme iron) dependent dioxygenase (DOX), which enzymatically forms a hydroperoxy fatty acid, and a subsequent rearrangement of this intermediate by a class III cytochrome P450 [5]. Whereas many aspects of oxylipin biosynthesis in plants are characterized, an emerging field of oxylipin research focuses on the exploration of oxylipin diversity and biosynthesis in fungi [1]. One important class of fungal oxylipins has been named due to its involvement in regulation of the sexual and asexual life cycle of the fungus: precocious sexual inducers (Psi-factors) [6-8]. These fungal oxylipins are of particular interest, since they have not only been proposed to mediate developmental processes of the fungus, but also to be involved in host-pathogen communication and thus potentially promote fungal infections of plants and mammals $[1,3,9,10]$. The enzymes responsible for biosynthesis of these Psi-factors were initially identified in Aspergillus nidulans as orthologs of 7,8-linoleate diol synthase (7,8-LDS) from Gaeumannomyces graminis and have been named Psi-factor producing oxygenases (Ppo) [11-13]. Whereas at this time 7,8-LDS was thought to be functionally [14] and structurally [15] similar to the mammalian prostaglandin H2-synthase (PGHS), bioinformatic [12,16,17] and biochemical [17] analyses of the prototype enzyme of the new Ppo-family, PpoA, revealed that the protein 
has two independent heme domains. The N-terminal domain, which also shows structural and functional homology to PGHS, inserts molecular oxygen at the allylic $C_{8}$ of an unsaturated C18 fatty acid. This hydroperoxy derivative serves then as substrate for the C-terminal domain. Here, a class III cytochrome P450, which neither requires external reducing equivalents nor catalyzes substrate monooxygenations, rearranges the 8-hydroperoxy fatty acid (e.g. 8-hydroperoxy octadecadienoic acid; 8-HPODE) to its 5,8-dihydroxy derivative (e.g. 5,8-dihydroxy octadecadienoic acid; 5,8-DiHODE). Notably, this twostep process of oxylipin biosynthesis, with lipid peroxidation by a DOX and subsequent rearrangement of the hydroperoxy fatty acid intermediate by an atypical cytochrome P450, resembles the typical oxylipin biosynthesis pathways of mammals and plants (Fig. 1). Later it was shown that the previously described 7,8-LDS exhibits a similar domain architecture with its $\mathrm{P} 450$-domain possessing a distinct specificity and rearranging the intermediately formed 8-hydroperoxy fatty acid to its 7,8-dihydroxy derivative [18]. Several studies could confirm the proposed homology of the distinct PpoA domains to oxylipinforming enzymes from a mechanistic $[19,20]$ as well as from a structural point of view [15,21]. For example, Fielding et al. could confirm that fatty acid dioxygenation by PpoA involves a tyrosyl radical [20], which may possess the same function as the catalytic competent tyrosine of PGHS [22]. Although sequence alignments between these two enzymes suggested that the tyrosyl radical is formed at Tyr374 in PpoA, a recent structural model of PpoA's DOX-domain raised the possibility that Tyr327 might serve as terminal site within a radical propagation chain [21]. In addition, an arginine-residue that may be involved in ionic binding of the fatty acid substrate within the DOX-domain was identified. On the other hand, affinity of the hydroperoxy fatty acid derivative to the cytochrome P450-domain in the next step of the reaction is not governed by ionic interactions and no determinants of substrate binding to this domain have been identified to date.

In contrast to the reaction mechanism of Ppo enzymes, knowledge of their structure and especially of the arrangement of the two catalytic domains within a single protein is still scarce. Here, we addressed this question by small-angle X-ray scattering (SAXS) in combination with atomic structure prediction of the single domains described previously [21]. The obtained model was validated by limited proteolysis of the protein. Based on this model and newly measured double electron-electron resonance (DEER) data for the wild type enzyme as well as for the previously described Tyr327Phe variant, we conclude that Tyr327 does not serve as an alternative radical site. Furthermore, we utilized the derived enzyme model to get further insights into the substrate binding mode of the cytochrome P450-domain of PpoA.

\section{Materials and methods}

\subsection{Materials}

Unless stated otherwise, all chemicals were obtained from Carl-Roth (Karlsruhe, Germany) and were of highest quality available. PfuPolymerase and DpnI were purchased from Fermentas (St. Leon-Rot, Germany) and peroxide-free fatty acids were obtained from Cayman Chemical (Ann Arbor, Michigan, USA).

\subsection{Methods}

\subsubsection{Enzyme expression and purification}

Enzyme expression and purification were performed as described by Brodhun et al. [17] with some slight modifications: The addition of $5 \mathrm{mM}$ EDTA to the buffer (50 mM Tris/HCl (pH 7.6) containing 10\% glycerol) used for the first anion exchange chromatography step enabled us to generate pure PpoA (>90\%) with the following size exclusion chromatography step (using $50 \mathrm{mM}$ Tris/ $\mathrm{HCl}$ (pH 7.6) as mobile phase). With this modification we were able to omit the additional anion exchange chromatography step reported before [17]. The protein-concentration was determined by UV-absorption utilizing a specific absorption coefficient $\varepsilon_{280}=125.500 \mathrm{M}^{-1} \mathrm{~cm}^{-1}$ and a molecular weight of 120.784 Da per monomer. Both parameters were calculated from PpoA's proteinsequence (Uniprot-ID: Q6RET3) by the ProtParam-algorithm (http:// web.expasy.org/protparam/).

\subsubsection{SAXS: measuring and modeling}

Synchrotron X-ray scattering data of the wild type enzyme were collected at the EMBL X33 beamline (DESY, Hamburg) [23] using a
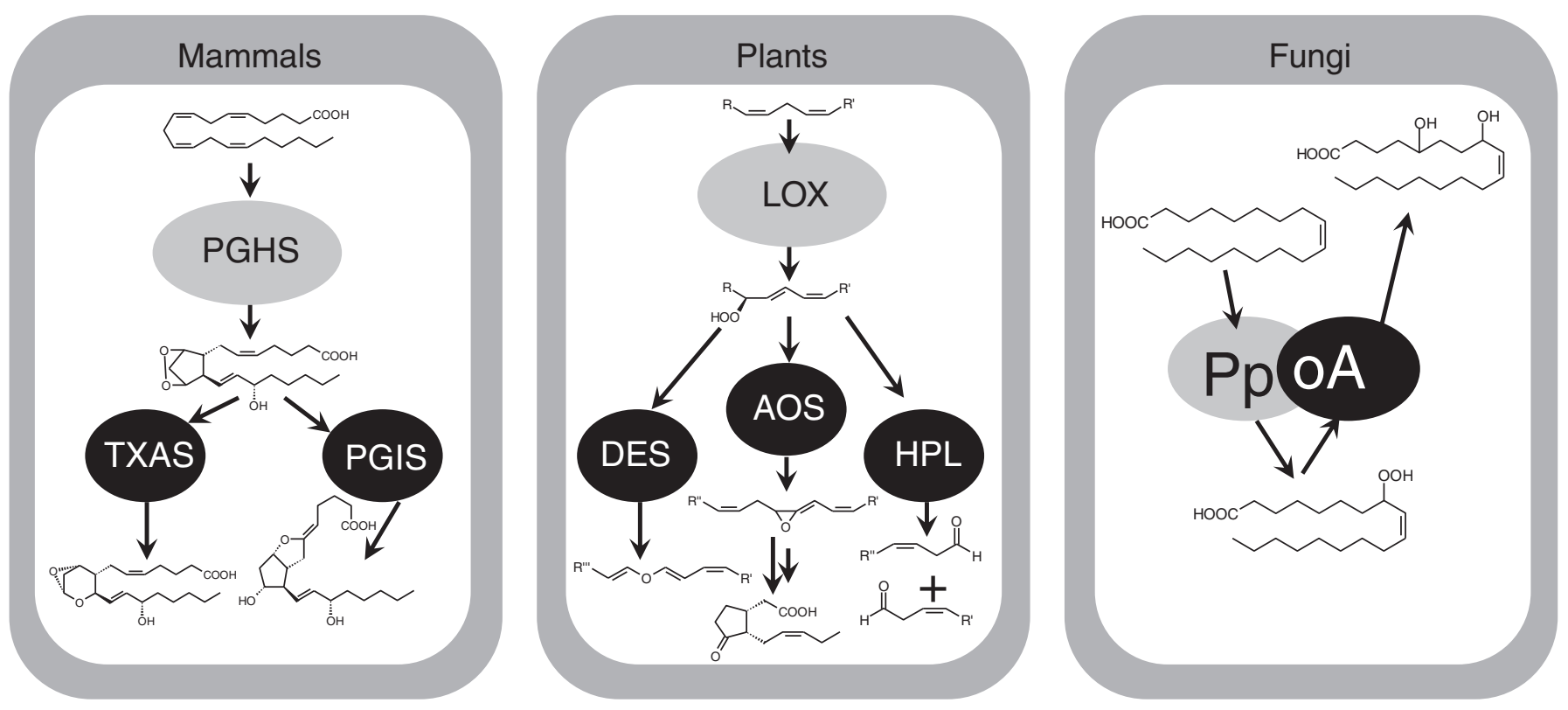

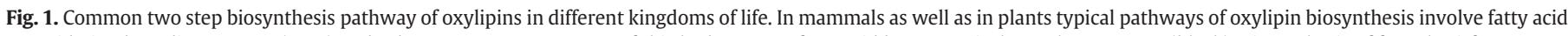

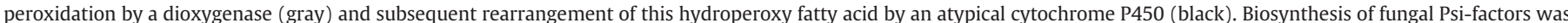

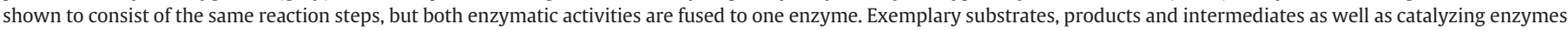

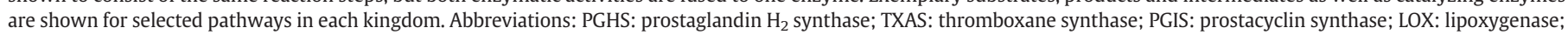
DES: divinyl ether synthase; AOS: allene oxide synthase; HPL: hydroperoxide lyase; PpoA: Psi-factor producing oxygenase A. 
robotic sample changer [24]. PpoA wild type enzyme was prepared in 20 mM HEPES, pH 7.4; 2 mM DTT was added facultatively and these preparations were measured at several solute concentrations in a range from 26 to $0.4 \mathrm{~g} / \mathrm{l}(26.0 \mathrm{~g} / \mathrm{l}, 9.8 \mathrm{~g} / \mathrm{l}, 5.0 \mathrm{~g} / \mathrm{l}, 2.1 \mathrm{~g} / \mathrm{l}, 1.1 \mathrm{~g} / \mathrm{l}, 0.6 \mathrm{~g} / \mathrm{l}$ and $0.4 \mathrm{~g} / \mathrm{l})$. SAXS data were recorded on a PILATUS $1 \mathrm{M}$ pixel detector (DECTRIS, Baden, Switzerland) at a sample-detector distance of $2.7 \mathrm{~m}$ and a wavelength of $1.5 \AA$. This set up covers a range of momentum transfer of $0.012<s<0.6 \AA^{-1}(s=4 \pi \sin (\theta) / \lambda$, where $2 \theta$ is the scattering angle). The measured data were processed by PRIMUS [25]. The forward scattering $I(0)$ and the radius of gyration $\left(R_{g}\right)$ were calculated using the Guinier approximation [26] and assuming that at very small angles $\left(s<1.3 / R_{g}\right)$ the intensity is represented as $I(s)=$ $I(0) * \exp \left(-\left(s R_{g}\right)^{2} / 3\right)$. The pair-distance distribution function $p(r)$, from which the maximum particle dimension $\left(D_{\max }\right)$ is estimated, was computed using GNOM [27]. Finally, the excluded volume of the hydrated particle was computed using the Porod invariant [28] and the molecular mass was estimated as 0.6 times the Porod volume.

Low resolution shape analysis of the solute was performed using the ab initio program DAMMIF [29]. This algorithm represents the macromolecule by an assembly of densely packed beads and employs simulated annealing to build a compact interconnected configuration of beads inside a sphere with a diameter $\left(D_{\max }\right)$ that fits the experimental data $I_{\exp }(s)$ and minimizes the discrepancy $\chi^{2}$ :

$x^{2}=\frac{1}{N-1} \sum_{j=1}^{N}\left[\frac{I_{\exp }\left(s_{j}\right)-c I_{\text {calc }}\left(s_{j}\right)}{\sigma\left(s_{j}\right)}\right]^{2}$

where $N$ is the number of experimental points, $c$ is a scaling factor and $I_{\text {calc }}(s)$ and $\sigma\left(s_{j}\right)$ are the calculated intensity and the experimental error at the momentum transfer $s_{j}$, respectively. Several independent simulated annealing runs were performed and the results were analyzed using DAMAVER [30]. Predicted high resolution structures of PpoA's DOX- and P450-domain [21] were used as input for molecular docking. The program SASREF [31] was then employed for rigid body modeling. SASREF performs quaternary structure modeling of a complex formed by subunits with known atomic structure against solution scattering data minimizing the discrepancy $\chi^{2}$ between the experimental and calculated data (Eq. (1)). In order to make a feasible model, the N-terminal of the P450-domain was kept at a distance of $4 \AA$ from the C-terminal of the DOX-domain. The final solution is free of steric clashes. Finally, this model was super-imposed on the ab initio model using the program SUPCOMB [32]. All programs used for analysis of the SAXS data belong to the ATSAS package [33].

\subsubsection{Tryptic-digest of native PpoA and Peptide-assignment by LC/MS ${ }^{2}$}

Purified wild-type enzyme in $20 \mathrm{mM}$ HEPES buffer ( $\mathrm{pH}$ 7.4) was treated with $1: 200(\mathrm{w} / \mathrm{w})$ trypsin. After the reaction was accomplished over night at $4{ }^{\circ} \mathrm{C}$, the peptide-pattern was analyzed by $10 \%$ SDS-PAGE. Complete in-gel digestion of fragments from the SDS-PAGE was performed according to Shevchenko et al. [34]. Peptides were separated on an RSLCnano Ultimate 3000 system (Thermo Scientific, Waltham, USA). Therefore, peptides were bound on an Acclaim ${ }^{\circledR}$ PepMap 100 column (75 mm $\times 2$ cm, C18, 3 mm, $100 \AA$ A P/N164535 Thermo Scientific) and washed for $12 \mathrm{~min}$ with $4 \mu \mathrm{l} / \mathrm{min} 0.05 \%$ trifluoroacetic acid. Analytical peptide separation by reverse phase chromatography was performed on an Acclaim ${ }^{\circledR}$ PepMap RSLC column $(75 \mu \mathrm{m} \times 15 \mathrm{~cm}, \mathrm{C} 18$, $3 \mu \mathrm{m}, 100 \AA \AA \mathrm{A} / \mathrm{N} 164534$ Thermo Scientific) running a gradient from $3.2 \%$ acetonitrile $/ 0.1 \%$ formic acid to $40 \%$ acetonitrile $/ 0.1 \%$ formic acid within $25 \mathrm{~min}$ at a flow rate of $250 \mathrm{nl} / \mathrm{min}$. Nano ESI mass spectrometry on Orbitrap Velos Pro (Thermo Scientific): Peptides eluting from this column were on-line ionized by nano-electrospray (nESI) using the Nanospray Flex Ion Source at $2.4 \mathrm{kV}$ and continuously transferred into the mass spectrometer. Full scans within $\mathrm{m} / \mathrm{z}$ of $300-1850$ were recorded by the Orbitrap-FT analyzer at a resolution of 30.000 (using $\mathrm{m} / \mathrm{z}=445.120025$ as lock mass) with parallel data-dependent top 10 $\mathrm{MS}^{2}$-fragmentation in the LTQ Velos Pro linear ion trap. MS/MS ${ }^{2}$ data processing for protein analysis and identification was done with the Proteome Discoverer 1.3 software (Thermo Scientific) using the Sequest analysis algorithm and a taxon-defined protein database.

\subsubsection{Double-Electron Electron Resonance (DEER)}

The tyrosyl radical was generated by activating $300 \mu \mathrm{M}$ of PpoAmonomer with an equal volume of $16 \mathrm{mM}$ 8-HPODE in $50 \mathrm{mM}$ HEPES, pH 7.5, containing 30\% glycerol. Upon mixing, the reaction was transferred to a Q-band ( $34 \mathrm{GHz}$ ) EPR tube ( $2 \mathrm{~mm}$ outer diameter) and manually quenched after 5 to $7 \mathrm{~s}$ by freezing in liquid nitrogen. Pulsed EPR-measurements were performed on a Bruker ELEXSYS E580 equipped with Q-band capabilities. Temperature was adjusted using a liquid helium cryostat CF935 from Oxford Instruments (Abingdon, UK). For optimal sensitivity, the measurements were performed at $5 \mathrm{~K}$. DEER data were collected utilizing a dead-time free four-pulse sequence [35] and the parameters of the pulse-sequence were typically set as follows: $\pi / 2=20 \mathrm{~ns} ; \tau_{1}=200 \mathrm{~ns} ; \pi($ PELDOR $)=50 \mathrm{~ns}$ and the shot repetition time was set to $100 \mathrm{~ms}$. A two-step phase cycle was used to suppress offset contributions to the modulation depth. Distance distributions were extracted from dipolar time evolution traces using fitting with Tikhonov regularization as implemented in DeerAnalysis2011 [36].

\subsubsection{Site-directed mutagenesis}

Site-specific nucleotide exchanges were conducted on the previously described expression plasmid [17] by utilization of oligonucleotides with specific mismatches at the desired position. Details of the reaction are specified in [21] and the sequences of the utilized oligonucleotides are given in the supplemental material (Table S1). Insertion of site-specific mutations was confirmed by sequencing of the entire open reading frame. The plasmid encoding the Tyr327Phe variant of PpoA was constructed in a previous study [21].

\subsubsection{Product identification by $L C / M S^{2}$ and UHPLC ESI QTOF MS/MS analysis}

PpoA wild type and variants were expressed in three independent batches. After cell disruption, the lysate was incubated with $100 \mu \mathrm{M}$ peroxide-free oleic acid and linoleic acid, respectively. The reaction was stopped and reaction products were extracted after 2 min incubation time by $\mathrm{Et}_{2} \mathrm{O}$. $\mathrm{LC} / \mathrm{MS}^{2}$-analysis was performed as described previously [17]. Products were assigned based on previously reported spectra. The respective spectra can be found in the following publications: [37] (8,11-DiHODE); [38] (5,8-DiHODE); [19] (6,8-DiHOME); [39] (8-hydroxy-9,10-epoxy-12Z-octadecenoic acid); [40] (10-hydroxy-8,9-epoxy-12Z-octadecenoic acid); [38] (8-HODE); [39] (8-KODE).

For fragmentation studies the samples were analyzed by Ultra High Performance Liquid Chromatography (1290 Infinity, Agilent Technologies, Santa Clara, USA) coupled with a 6540 UHD Accurate-Mass QTOF LC MS instrument with Agilent Jet Stream Technology as ESI source (Agilent Technologies, Santa Clara, USA). For LC a ACQUITY UPLC BEH SHIELD RP18 column $(1 \times 100$ mm, $1.7 \mu$ m particle size, Waters Corporation, Milford, USA) was used at a temperature of $40{ }^{\circ} \mathrm{C}$ and a flow rate of $0.5 \mathrm{ml} / \mathrm{min}$. Water was used as solvent $A$ and acetonitrile as solvent $B$. $0.1 \%$ formic acid was added to both solvents. The oxylipin species were eluted under the following conditions: a gradient from 40 to $100 \%$ solvent B in $8 \mathrm{~min}$ and 100\% solvent B for $4 \mathrm{~min}$. The column was re-equilibrated at $40 \%$ solvent B for $4 \mathrm{~min}$.

The QTOF MS was operated in the targeted MS/MS mode with a detection frequency of $4 \mathrm{GHz}$. The source conditions were as follows: gas temperature: $250{ }^{\circ} \mathrm{C}$; drying gas flow: $81 \mathrm{~min}^{-1}$; nebulizer pressure: $35 \mathrm{psi}$; sheath gas temperature: $300{ }^{\circ} \mathrm{C}$; sheath gas flow: $81 \mathrm{~min}^{-1}$; VCap voltage: $3.0 \mathrm{kV}$; nozzle voltage: $200 \mathrm{~V}$; fragmentor voltage: $120 \mathrm{~V}$. All samples were ionized in negative ionization mode. Precursor 
ions were isolated within the narrow isolation width of $\sim 1.3 \mathrm{~m} / \mathrm{z}$. For exact mass measurement the reference mass correction with trifluoroacetic acid $\left([\mathrm{M}-\mathrm{H}]^{-} 112.98559\right)$ and HP-921 ([M $\left.+\mathrm{HCO}_{2}\right]^{-}$ 966.00073) as reference compounds was used. The LC MS was operated under Mass Hunter B04.00. Data were acquired by Mass Hunter Workstation Acquisition software B.04.00 (Agilent Technologies, Santa Clara, USA). Mass Hunter Qualitative Analysis B.05.00 (Agilent Technologies, Santa Clara, USA) was used for data analysis. The MS/MS spectra of the oxylipins were compared with the fragment information of the authentic standards and/or data from the literature.

\subsubsection{X-band EPR}

Continuous wave spectra of PpoA-variants at X-band were measured as described in our previous studies $[20,21]$.

\section{Results}

\subsection{SAXS}

In order to answer the question, how the two catalytic domains are arranged in PpoA, we performed SAXS-measurements in combination with atomic structure prediction of the single domains as described previously [21]. The size-exclusion column used for protein purification assured sample's monodispersity, which is a prerequisite for SAXS-measurements. Radiation damage during X-ray exposure was monitored by several successive measurements of a single protein solution. No significant changes were observed (data not shown). The scattering curves obtained for enzyme-concentrations from 0.5 to $10 \mathrm{~g} / \mathrm{l}$ showed very little concentration dependency (Supplemental material, Fig. S1) and the data were extrapolated to infinite dilution using standard methods. The overall parameters derived from the extrapolated curve displayed in Fig. 2 are presented in Table 1. Based on the Porod volume, $M_{r}$ was determined to be $\sim 315 \mathrm{kDa}$, which corresponds well to the expected value for a trimeric protein $(330 \mathrm{kDa})$. The $p(r)$ function had a bell shape typical for globular proteins and revealed $D_{\max }$ of $16.5 \pm 1 \mathrm{~nm}$. Twenty independent runs of DAMMIF computed without symmetry restrictions were averaged by DAMAVER, providing the $M_{r}$ estimate of $\sim 330 \mathrm{kDa}$, which is also compatible with a trimeric quaternary structure. Therefore, DAMMIF was also run with P3 symmetry. Again, 20 independent runs were performed and averaged by DAMAVER. The reconstruction assuming P3 symmetry is similar in the overall shape to that in $\mathrm{P} 1$ but the symmetric one provided more structural detail (Fig. 3A, B). This result is supported by an independent determination

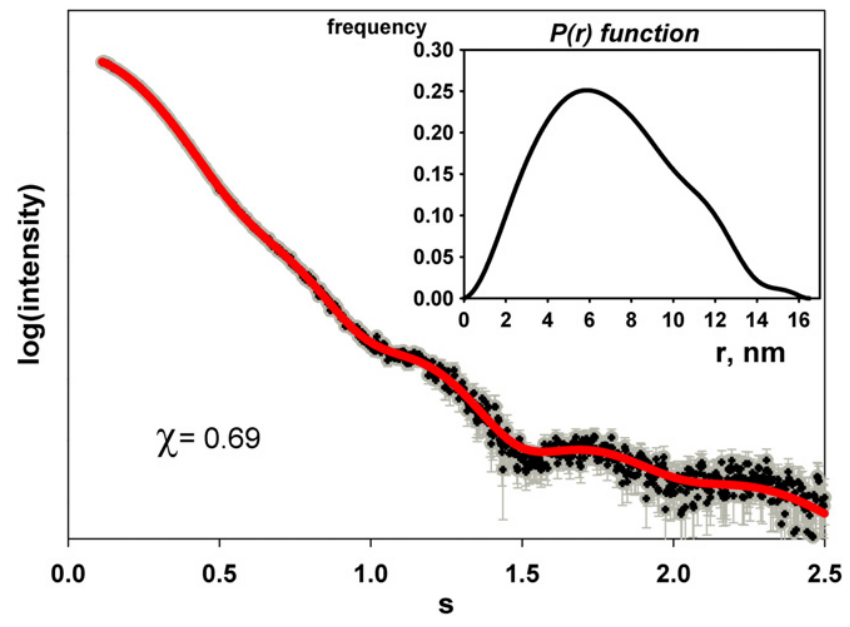

Fig. 2. Experimental SAXS data vs. theoretical scattering (red) using SASREF. The figure also shows the derived pair-distance distribution function $p(r)$.
Table 1

Data collection and scattering derived parameters for PpoA wild type enzyme. Abbreviations: $M_{r}$ : molecular mass; $R_{g}$ : radius of gyration; $D_{\max }$ : maximal particle dimension; $V_{p}$ : Porod volume; $V_{e x}$ : Particle excluded volume.

\begin{tabular}{|c|c|}
\hline Instrument & $\begin{array}{l}\text { EMBL X33 beam line storage } \\
\text { ring DORIS III (DESY, Hamburg) }\end{array}$ \\
\hline \multicolumn{2}{|l|}{ Data collection parameters } \\
\hline Beam geometry & $2 \times 0.6 \mathrm{~mm}^{2}$ \\
\hline Wavelength $(\AA)$ & 1.5 \\
\hline$q$-Range $\left(\AA^{-1}\right)$ & $0.006-0.600$ \\
\hline Exposure time (min) & $2(8 \times 15 s)$ \\
\hline Concentration range $\left(\mathrm{mg} \mathrm{ml}^{-1}\right)$ & $26-0.4$ \\
\hline Temperature $(\mathrm{K})$ & 283 \\
\hline \multicolumn{2}{|l|}{ Structural parameters } \\
\hline$I(0)$ (relative) (from $P(r)$ ) & $0.270 \pm 0.05$ \\
\hline$R_{g}(\AA)($ from $P(r))$ & $54.0 \pm 2$ \\
\hline$I(0)\left(\mathrm{cm}^{-1}\right)$ (from Guinier) & $0.270 \pm 0.05$ \\
\hline$R_{g}(\AA)($ from Guinier $)$ & $53.9 \pm 2$ \\
\hline$D_{\max }(\AA)$ & $165 \pm 10$ \\
\hline Porod volume estimate $\left(\AA^{3}\right)$ & $540,000 \pm 20,000$ \\
\hline Excluded volume estimate $\left(\AA^{3}\right)$ & $667,000 \pm 20,000$ \\
\hline Dry volume calculated from sequence $\left(\AA^{3}\right)$ & $\sim 648,000$ \\
\hline \multicolumn{2}{|l|}{ Molecular mass determination } \\
\hline $\begin{array}{l}\text { Molecular mass } M_{r}(\mathrm{Da}) \text { from Porod } \\
\quad \text { volume }\left(V_{p} * 0.6\right)\end{array}$ & $315,000 \pm 20,000$ \\
\hline $\begin{array}{l}\text { Molecular mass } M_{r}(\mathrm{Da}) \text { from excluded } \\
\quad \text { volume }\left(V_{e x} / 2\right)\end{array}$ & $330,000 \pm 20,000$ \\
\hline Calculated monomeric $M_{r}$ from sequence & $\sim 120,784$ \\
\hline \multicolumn{2}{|l|}{ Software employed } \\
\hline Primary data reduction & RADAVER \\
\hline Data processing & PRIMUS \\
\hline $\mathrm{Ab}$ initio analysis & DAMMIF \\
\hline Validation and averaging & DAMAVER \\
\hline Rigid body modeling & SASREF \\
\hline Computation of model intensities & CRYSOL \\
\hline 3D graphic representations & VMD \\
\hline
\end{tabular}

of the native enzyme's molecular weight by High Mass MALDI-TOF (Fig. S10).

In an independent modeling approach, the quaternary structure of PpoA was reconstructed by restrained rigid body refinement using SASREF and the predicted models of the two distinct PpoA-domains. The typical SASREF model provided a good fit to the experimental data with Chi $=0.69$ (Fig. 2) and displayed a nearly flat triangular shape (Fig. 3C, D), which was fully compatible with the ab initio reconstruction (Fig. 3E, F).

\subsection{Native trypsinolysis of PpoA and identification of accessible cleavage sites}

In order to verify this structural model, surface exposed protease cleavage sites were mapped. Therefore, native wild type enzyme was subjected to proteolysis with trypsin. This treatment did not alter the apparent native molecular weight as judged by size-exclusion chromatography (data not shown). However, upon denaturation four stable fragments were separated by SDS-PAGE (Fig. 4A). The sites that were accessible to proteolytic cleavage were identified by ESI-MS ${ }^{2}$ analysis of the excised bands. Therefore, the stable fragments derived from SDS-PAGE were completely in-gel trypsinated and the resulting peptides were analyzed by mass spectrometry. Using a semi-quantitative analysis, four accessible trypsin cleavage sites of the native protein can be suggested: Arg140, Lys350, Lys789 and Lys834 (Supplemental material, Fig. S2). These cleavage sites are highlighted within the predicted quaternary structure (Fig. 4B, blue spheres). Notably, the accessible digestion sites are distributed on the edge of the molecule and the flat sites of the particle are inaccessible for the protease's active site. 
A

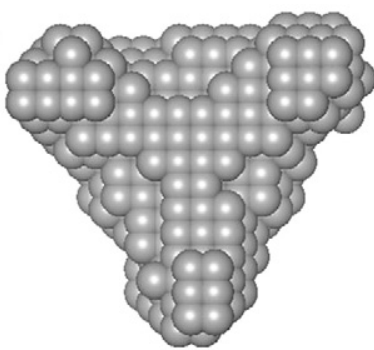

C

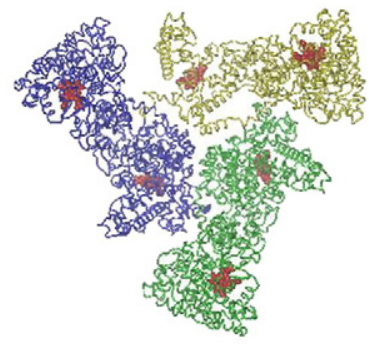

E

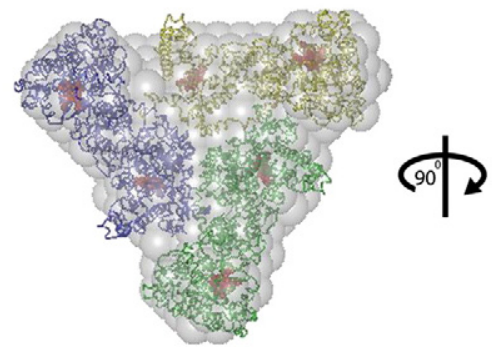

$\mathrm{B}$
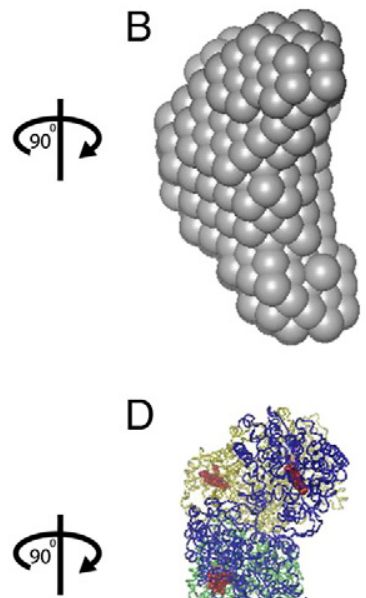

D

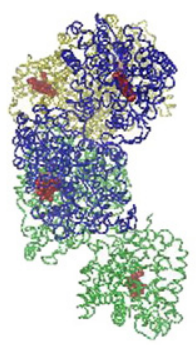

$\mathrm{F}$

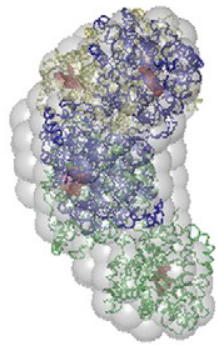

Fig. 3. PpoA's quaternary structure as derived from small-angle X-ray scattering. A. Ab initio determined bead-model of the particle shape that fits the experimentally observed scattering curve. B. $90^{\circ}$ rotation of A around the z-axis. C. Rigid-body docking of three PpoA-monomers (i.e. three predicted DOX- and three predicted P450-domains) to the scattering curve. D. $90^{\circ}$ rotation of $\mathrm{C}$ around the z-axis. E. Superimposition of A and C. F. Superimposition of B and D.
3.3. Measuring tyrosyl-tyrosyl distances by DEER and assigning the radical position

In the derived structural model of PpoA, the DOX-domains are arranged in the middle of the triangular structure, whereas the three cytochrome P450-domains are located at the vertices (Fig. 5, green vs. blue). This model gives rise to an equilateral distance triangle spanned between active site residues of the single monomers. As catalytic important residues of the DOX-domain two tyrosines are proposed to mediate fatty acid oxidation by a radical mechanism [17,20,21]. Within the structural model, the distances between these putative radical sites are $5.1 \pm 1 \mathrm{~nm}$ and $4.4 \pm 1 \mathrm{~nm}$, respectively (Fig. 5). Interestingly, a previous measurement of inter-spin distances at X-band EPR indicated that the observed tyrosyl radicals of the distinct monomers are spaced by a pair of distances [20]. Whereas the larger of these distances $(\sim 5.2 \mathrm{~nm})$ is in good agreement with the distances between the Tyr374s within the proposed trimeric structure, one may speculate that the shorter distance $(\sim 3.9 \mathrm{~nm})$ represents the distances between the Tyr327s and thus suggests an alternative radical at this site. To examine this hypothesis, the inter-spin distances in wild type enzyme were remeasured at Q-band, a frequency that provides higher spin sensitivity for DEER, and compared with the distance distribution measured for the Tyr327Phe variant. The results of this experiment revealed that the pair of distances previously obtained at X-band is reproducible also at Q-band (Fig. 6). Moreover, from the dipolar evolution traces of the Tyr327Phe variant basically the same distance distribution as for the wild type enzyme was extracted.

\subsection{Determinants of substrate binding to the cytochrome P450-domain of PpoA}

In contrast to the DOX-domain, ionic interactions with the substrate's carboxylate do not contribute to substrate binding to the cytochrome P450-domain of PpoA ([21] and Supplemental material). In the light of this finding, other determinants for substrate binding to the cytochrome P450-domain were sought. Assuming that the substrate is placed as
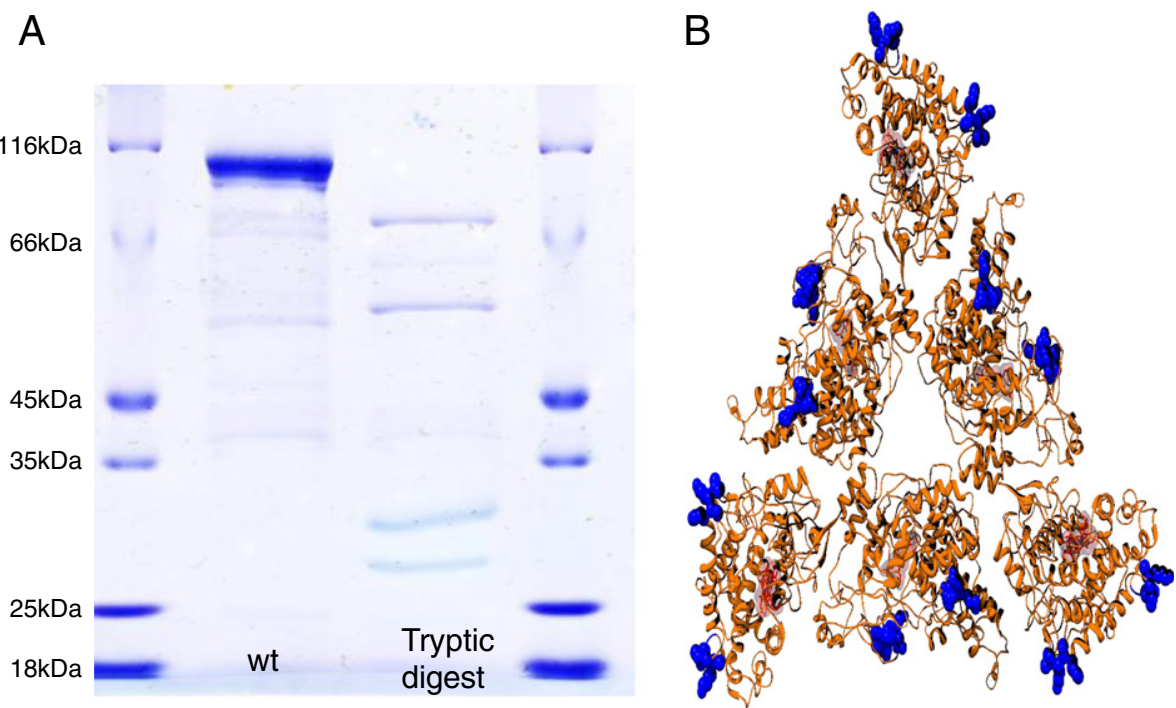

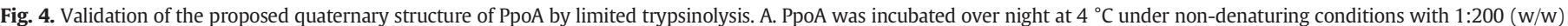

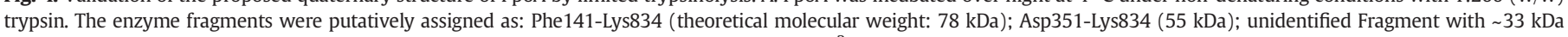

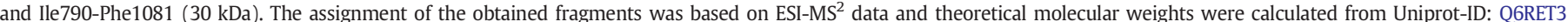

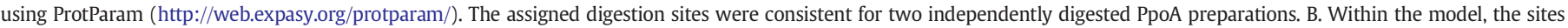

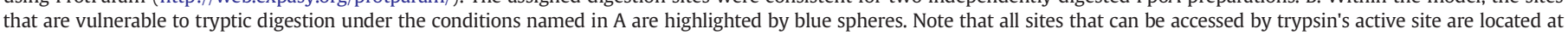
the edge of the protein and not on the flat side. 


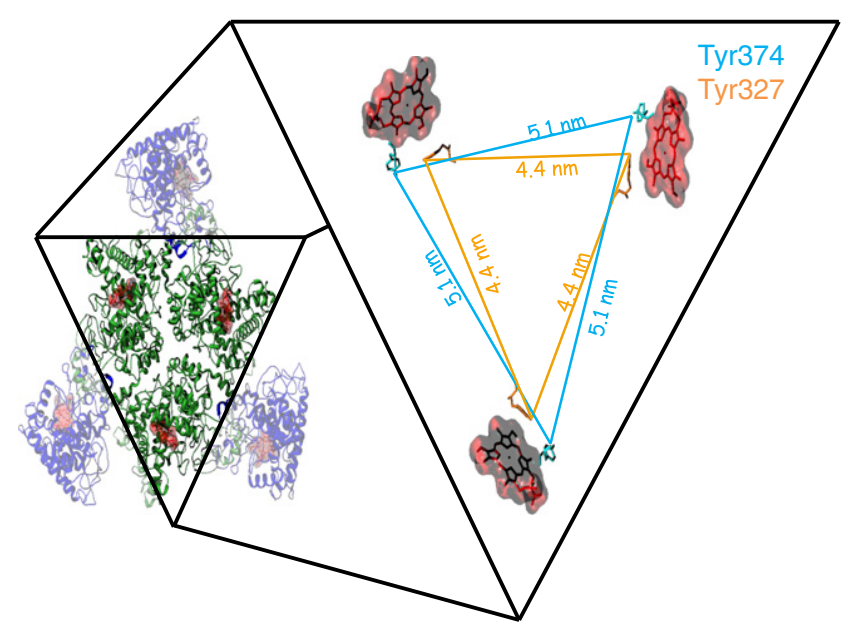

Fig. 5. Zoom into the DOX-Active sites of the SAXS-derived structural model. Heme cofactor and tyrosine residues putatively forming the substrate oxidizing radical are shown. The distances between the three Tyr374-sites (cyan) are $5.1 \mathrm{~nm}$, whereas the distances between the alternative Tyr327-sites (orange) are $4.4 \mathrm{~nm}$. Caused by the inherently low-resolution of SAXS, these distances are associated with an error of $\sim 1 \mathrm{~nm}$. Within the structural model of PpoA, DOX-domains are colored green and the P450-domains are colored blue. Hemes are rendered in red.

proposed before [21], a pair of phenylalanines (Phe795 and Phe799) is in proximity to the substrate's double-bonds and sequence alignments indicate that these phenylalanines are highly conserved in the Ppo enzyme family (Supplemental material, Figs. S3 and S4). Thus, one may assume that $\pi$-stacking between the phenyl ring of Phe795 and the $\Delta 9$ double bond and, optionally, between the phenyl ring of Phe799 and the $\Delta 12$ double bond of the substrate contributes to substrate binding and assures proper substrate placement. To experimentally assess the contributions of these active site residues to enzymatic turn-over, the respective leucine variants of PpoA (Phe795Leu and Phe799Leu) were generated by site-directed mutagenesis.

Since the phenyl ring of Phe795 was proposed to bind the $\Delta 9$ double bond of the substrate, oleic acid was utilized to probe the respective variant. Indeed, the product pattern of this conversion shows an increased amount of 6,8-DiHOME formed (Fig. 7A). This increased quantity of a positional isomer is consistent with Phe795 being involved in proper substrate placement. In contrast, conversion of linoleic acid leads to a smaller, but still significant, increase in the relative amount of 6,8 DiHODE (Supplemental material, Fig. S5). Considering that this substrate might be additionally constrained by its second double bond at $\Delta 12$, the contribution of the phenyl ring of Phe799 to substrate binding was analyzed next. Interestingly, conversion of linoleic acid by the Phe799Leu variant resulted in a significantly diversified product pattern as compared to wild type enzyme (Fig. 7B). The identity of the newly formed products (epoxy alcohols and ketones) suggested conversion of 8-HPODE by free heme as reported previously [40]. Indeed, the P450-heme of the Phe799Leu variant was found to be significantly less abundant than in wild type enzyme, but also for the purified enzyme the product pattern was diversified, which suggests that the loss of reaction specificity is not solely caused by reactivity of free heme (Fig. 8). To further investigate the mechanism by which Phe799 influences heme binding and reaction specificity, the respective tyrosine (Phe799Tyr), methionine (Phe799Met) and tryptophane (Phe799Trp) variants were constructed. Whereas the Phe799Tyr variant exhibited reaction specificity comparable to wild type enzyme, the bulky residue introduced in the Phe799Trp variant seemed to prevent substrate access and therefore 8-HPODE rearrangement and the Phe799Met variant exhibited basically the same diversified product pattern as the Phe799Leu variant (Supplemental material, Fig. S6). In contrast to the later, all other variants exhibited a UV/VIS-spectrum indicative of full heme occupancy (Supplemental material, Fig. S7). To finally assess, whether the thus shown effect of an aliphatic residue at position 799 on reaction specificity is due to $\pi$-stacking to the $\Delta 12$ double bond,
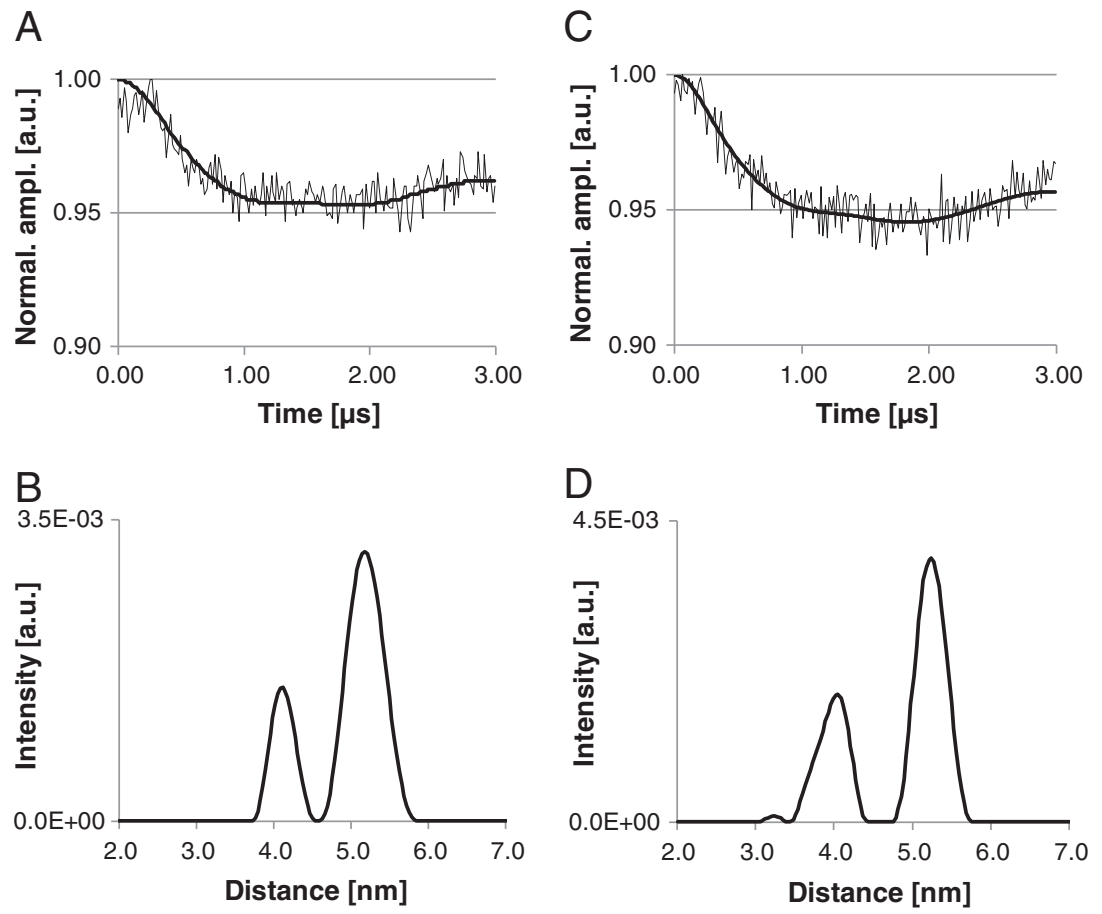

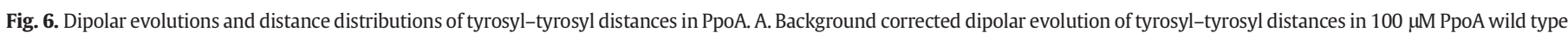

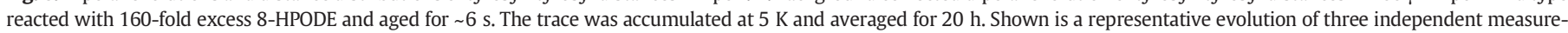

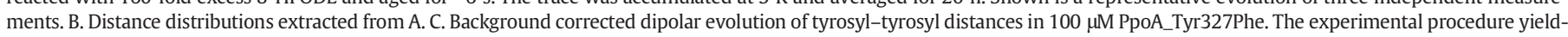
ing the radical is identical to the one specified for A. Shown is a representative evolution of two independent measurements. D. Distance distributions extracted from C. 

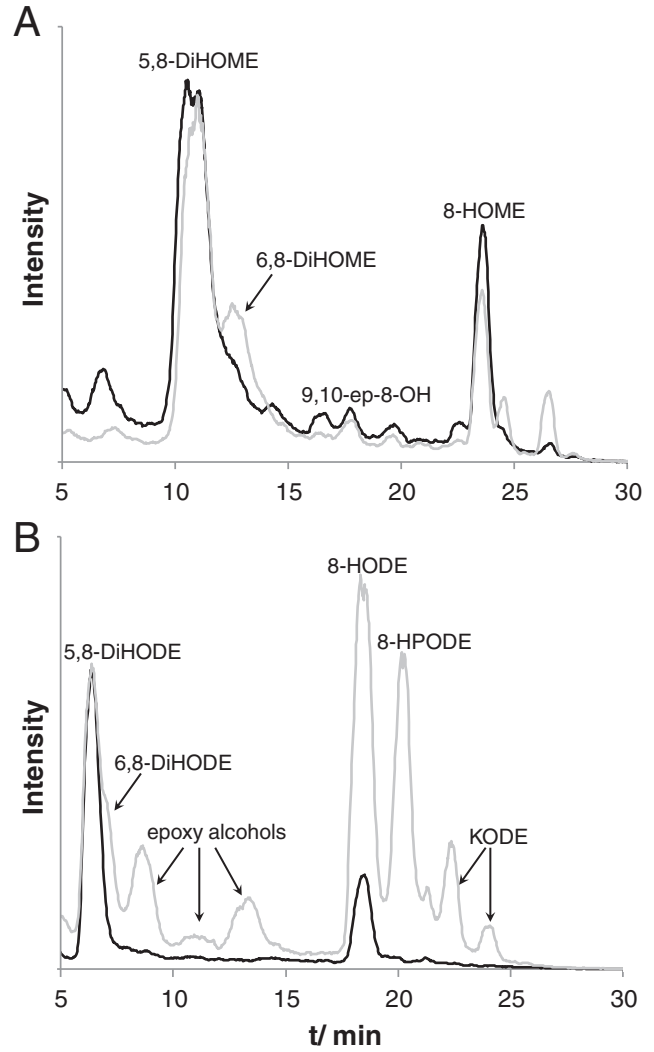

Fig. 7. Phe795 and Phe799 determine the reaction specificity of hydroperoxy fatty acid rearrangement catalyzed by PpoA. A. Conversion of oleic acid by wild type enzyme and the Phe795Leu variant. As previously reported [19], the main product of the wild type enzyme is 5,8-DiHOME. In contrast, the Phe795Leu variant forms a significantly increased amount of 6,8-DiHOME. Shown is a representative chromatogram from three biological replicates. Main products are marked. The unidentified products may be 8,11-DiHOME and 8-KOME. B. The variation of Phe799 to Leu provokes a severe diversification of the product pattern. Shown is a representative chromatogram for conversion of linoleic acid by three biological replicates. Abbreviations: DiHOME: dihydroxy-oleic acid; 9,10-ep-8OH: 8-hydroxy-9,10epoxy-stearic acid; HOME: hydroxy-oleic acid; KOME: oxo-oleic acid; DiHODE: dihydroxylinoleic acid; epoxy alcohols: 8-hydroxy-9,10-epoxy-12Z-octadecenoic acid and 10hydroxy-8,9-epoxy-12Z-octadecenoic acid; HODE: hydroxy-linoleic acid; HPODE: hydroperoxy-linoleic acid; KODE: oxo-linoleic acid.

conversions of oleic acid by the Phe799Leu variant were analyzed. This substrate lacks a $\Delta 12$ double bond and thus, if the reaction specificity would be based solely on $\pi$-stacking between the phenyl-ring of Phe799 and the substrate's $\Delta 12$ double bond, conversions of this substrate by the Phe799Leu-variant of PpoA should exhibit the same product pattern as the wild type enzyme. However, the product pattern was also diversified as compared to the wild type enzyme (Supplemental material, Fig. S8). Since the oxylipins derived from oleic acid are not as well characterized as the ones derived from linoleic acid, we identified those products by employing UHPLC ESI QTOF MS/MS analysis as shown in Supplemental material Table S2 and Fig. S9. This method provides not only a better separation of the formed metabolites but also exact mass information of the MS/MS-fragments, which facilitates the unequivocal identification of products. Interestingly, this analysis showed that among the formed products diastereomers of the 5,8-DiHOME derivative exist. These have also been observed before [19]. In addition we found 8,11-DiHOME, 6,8-diHOME, two diastereomers of the respective epoxyalcohols (tentatively assigned as 9,10-Ep-8-HOME and 8,9-Ep10-HOME according to [40]) as well as 8-HOME and 8-KOME.

\section{Discussion}

Based on results from size-exclusion chromatography, which indicated that PpoA and related enzymes have an apparent molecular mass of approx. 440-520 kDa, it was suggested that these enzymes consist of four subunits building a tetrameric protein complex $[14,17]$. The data obtained in the present study by SAXS experiments reveal that this view on the molecular weight was not precise enough. Moreover, this result is supported by an independent determination of the native enzyme's molecular weight by High Mass MALDI-TOF (Fig. S10) and may be explained by the proposed, relatively flat, trimeric quaternary structure (Fig. 3). This flat shape may cause an error prone estimation of the native molecular weight, if it is determined by size exclusion chromatography, which is calibrated for globular proteins. Based on docking of previously derived structural models of the distinct PpoA domains [21] to the scattering curve, a quaternary structure with atomic resolution is proposed. This structure may reasonably explain why tryptic digestion of native PpoA does not lead to its complete degradation (Fig. 4). The positions of the identified accessible cleavage sites within the structural model suggest that only the edges of the flat molecule can reach into the active site of trypsin, whereas potential cleavage sites on the flat surfaces of PpoA are inaccessible.

With the atomic structural model of the PpoA trimer on hand, the catalytic competent active site tyrosyls in the DOX-domain were examined next. Homology between the active site architecture of PpoA and that of PGHS suggested that two tyrosine residues may serve as radical sites: Tyr374 and Tyr327 [15,21]. The distances between these residues in the here proposed model and two tyrosyltyrosyl distances extracted from a previous DEER-measurement [20] indicated that a radical might be formed at both active site tyrosines. Whereas the longer distance of $5.2 \mathrm{~nm}$ matched the distance between the three Tyr374 of the trimeric complex of our model (Fig. 5), the shorter distance was initially suggested to arise from dipolar couplings of amino acid radicals formed at Tyr327. Surprisingly, however, our DEER analysis of the respective Tyr327Phe-variant resulted in the same pair of distances (4.1 and $5.2 \mathrm{~nm}$, respectively; Fig. 6) with basically the same intensity distribution as that of the wild type enzyme. This smaller distance may reflect a non-physiological artifact caused by the high enzyme-concentrations needed for this kind of measurements. In fact, SAXS-analysis has identified particle repulsion at concentrations $>26 \mathrm{~g} / \mathrm{l}$ suggesting polydispersity of the enzyme at high concentration (data not shown). This might imply that the smaller distance measured by DEER, represents the distance between the catalytic tyrosyls in one of the alternative oligomers formed at high concentrations in vitro. To additionally strengthen this hypothesis, we also evaluated some alternative scenarios potentially causing this smaller distance. However, we finally excluded most of them or judged them to be unlikely (cf. Supplemental Table S3).

Moreover, the proposed atomic structure was utilized to investigate substrate binding to PpoA's P450 active site. Two phenylalanines (Phe795 and Phe799) in proximity to the substrate's double bonds suggested that $\pi$-stacking may play a role in this respect. In line with such a mechanism, previous reports suggested that regioselectivity of hydroperoxide rearrangement is rather determined by the distance from the substrate's double bonds than by the distance to the fatty acid's $\omega$ or carboxyl end [17,41]. Interestingly, the two identified phenylalanines are located in the substrate recognition sites 2 and 3, i.e. between the $\alpha$-helices F and G. Since substrate recognition sites are generally considered to determine substrate binding and reaction specificity of cytochrome P450s [42], the identified two phenylalanines may possess a similar function in PpoA. In the proposed model, however, substrate bound to Phe795 and Phe799 may be too distant from heme, to be in a catalytic active position. Nevertheless, for cytochrome P450s conformational changes upon substrate binding are common and may favorably affect the F- and G-helices, which may lead to a closure of the active site $[43,44]$. Considering this conformational flexibility of cytochrome P450s, the identified phenylalanines are reasonable determinants for substrate binding. Consequently, interactions between the substrate and Phe795 and Phe799, respectively, would constrain the 
A

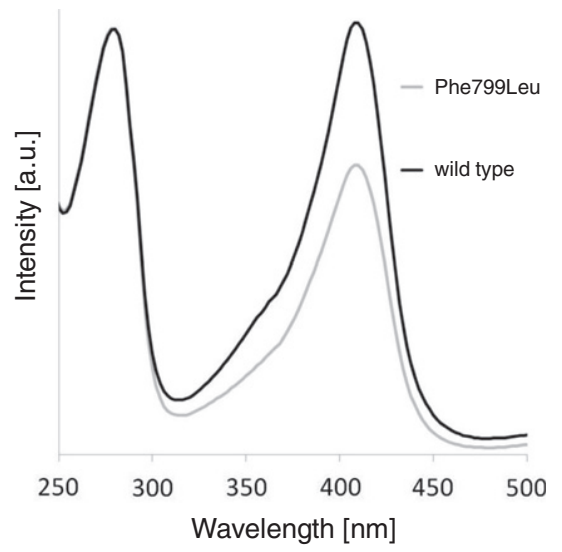

C

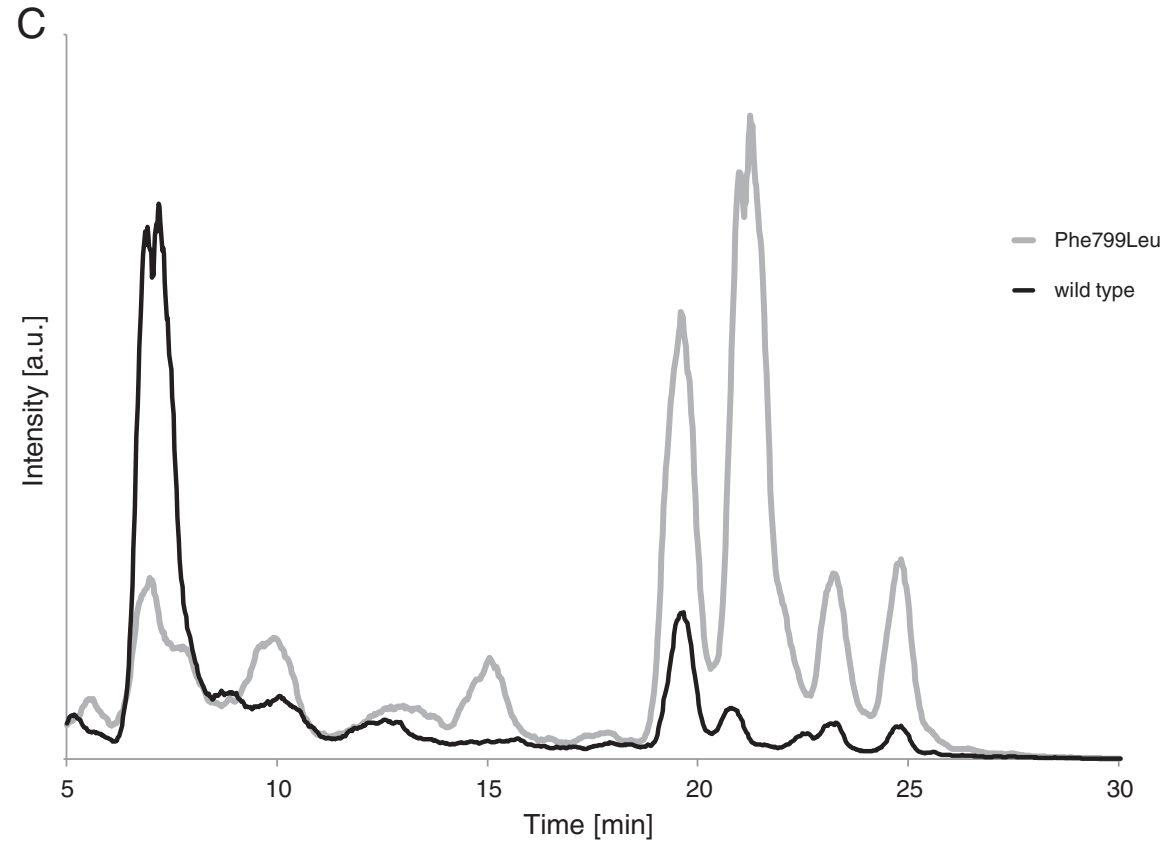

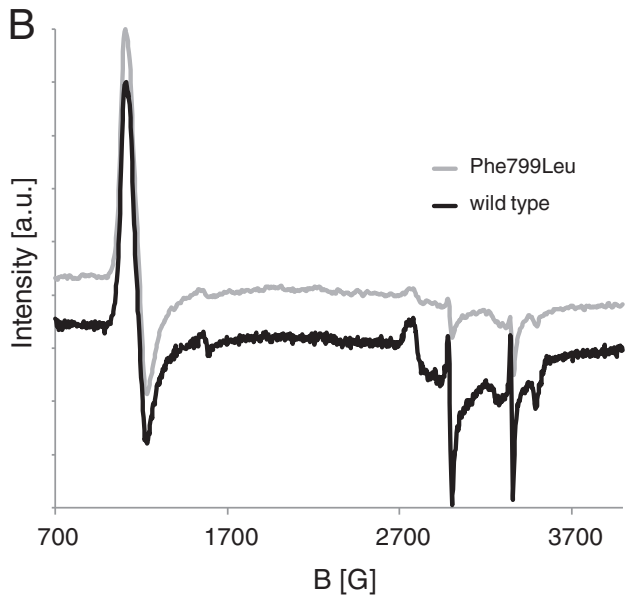


of two phenylalanines. Furthermore, the assignment of Tyr374 as solely catalytic competent tyrosine was strengthened by combining the SAXS-derived low resolution structure with the precise determination of tyrosyl-tyrosyl distances by DEER.

\section{Acknowledgement}

This work was financed by the German Research Foundation (DFG): INTERNATIONAL RESEARCH TRAINING GROUP 1422 Metal Sites in Biomolecules: Structures, Regulation and Mechanisms and the excellence initiative FL3 INST 186/822-1. We are grateful to Sabine Freitag for expert purification of 8-HPODE. In addition, CK was supported by the Biomolecules program of the Goettingen Graduate School of Neurosciences and Molecular Biology (GGNB). We are grateful to Dr. Gunnar Jeschke for discussing with us the origin of the $4.1 \mathrm{~nm}$ distance extracted from dipolar evolutions.

\section{Appendix A. Supplementary data}

Supplementary data to this article can be found online at http:// dx.doi.org/10.1016/j.bbalip.2013.06.003.

\section{References}

[1] F. Brodhun, I. Feussner, Oxylipins in fungi, FEBS J. 278 (2011) 1047-1063.

[2] A. Andreou, F. Brodhun, I. Feussner, Biosynthesis of oxylipins in non-mammals, Prog. Lipid Res. 48 (2009) 148-170.

[3] S.A. Christensen, M.V. Kolomiets, The lipid language of plant-fungal interactions, Fungal Genet. Biol. 48 (2011) 4-14

[4] C. Wasternack, B. Hause, Jasmonates: biosynthesis, perception, signal transduction and action in plant stress response, growth and development, An update to the 2007 review in Annals of Botany, Ann. Bot. 111, 2013, pp. 1021-1058.

[5] A.R. Brash, Mechanistic aspects of CYP74 allene oxide synthases and related cytochrome P450 enzymes, Phytochemistry 70 (2009) 1522-1531.

[6] S.P. Champe, P. Rao, A. Chang, An endogenous inducer of sexual development in Aspergillus nidulans, J. Gen. Microbiol. 133 (1987) 1383-1387.

[7] S.P. Champe, A.A. el-Zayat, Isolation of a sexual sporulation hormone from Aspergillus nidulans, J. Bacteriol. 171 (1989) 3982-3988.

[8] P. Mazur, K. Nakanishi, A.A.E. Elzayat, S.P. Champe, Structure and synthesis of sporogenic Psi factors from Aspergillus nidulans, J. Chem. Soc. Chem. Commun. 20 (1991) 1486-1487.

[9] D.I. Tsitsigiannis, J.W. Bok, D. Andes, K.F. Nielsen, J.C. Frisvad, N.P. Keller, Aspergillus cyclooxygenase-like enzymes are associated with prostaglandin production and virulence, Infect. Immun. 73 (2005) 4548-4559

[10] D.I. Tsitsigiannis, N.P. Keller, Oxylipins act as determinants of natural product biosynthesis and seed colonization in Aspergillus nidulans, Mol. Microbiol. 59 (2006) 882-892.

[11] D.I. Tsitsigiannis, T.M. Kowieski, R. Zarnowski, N.P. Keller, Three putative oxylipin biosynthetic genes integrate sexual and asexual development in Aspergillus nidulans, Microbiology 151 (2005) 1809-1821.

[12] D.I. Tsitsigiannis, R. Zarnowski, N.P. Keller, The lipid body protein, PpoA, coordinates sexual and asexual sporulation in Aspergillus nidulans, J. Biol. Chem. 279 (2004) 11344-11353.

[13] U. Garscha, F. Jerneren, D. Chung, N.P. Keller, M. Hamberg, E.H. Oliw, Identification of dioxygenases required for Aspergillus development: studies of products, stereochemistry, and the reaction mechanism, J. Biol Chem 282 (2007) 34707-34718.

[14] C. Su, M. Sahlin, E.H. Oliw, A protein radical and ferryl intermediates are generated by linoleate diol synthase, a ferric hemeprotein with dioxygenase and hydroperoxide isomerase activities, J. Biol. Chem. 273 (1998) 20744-20751.

[15] U. Garscha, E.H. Oliw, Critical amino acids for the 8(R)-dioxygenase activity of linoleate diol synthase. A comparison with cyclooxygenases, FEBS Lett. 582 (2008) 3547-3551.

[16] D.-S. Lee, P. Nioche, M. Hamberg, C.S. Raman, Structural insights into the evolutionary paths of oxylipin biosynthetic enzymes, Nature 455 (2008) 363-368.

[17] F. Brodhun, C. Gobel, E. Hornung, I. Feussner, Identification of PpoA from Aspergillus nidulans as a fusion protein of a fatty acid heme dioxygenase/peroxidase and cytochrome P450, J. Biol. Chem. 284 (2009) 11792-11805.
[18] I. Hoffmann, F. Jernerén, U. Garscha, E.H. Oliw, Expression of 5,8-LDS of Aspergillus fumigatus and its dioxygenase domain. A comparison with 7,8-LDS, 10-dioxygenase, and cyclooxygenase, Arch. Biochem. Biophys. 506 (2011) 216-222.

[19] A. Nadler, C. Koch, F. Brodhun, J.-D. Wehland, K. Tittmann, I. Feussner, U. Diederichsen, Influence of substrate dideuteration on the reaction of the bifunctional heme enzyme Psi factor producing oxygenase A (PpoA), ChemBioChem 12 (2011) 728-737.

[20] A.J. Fielding, F. Brodhun, C. Koch, R. Pievo, V. Denysenkov, I. Feussner, M. Bennati, Multifrequency electron paramagnetic resonance characterization of $\mathrm{PpoA}$, a CYP450 fusion protein that catalyzes fatty acid dioxygenation, J. Am. Chem. Soc. 133 (2011) 9052-9062.

[21] C. Koch, A.J. Fielding, F. Brodhun, M. Bennati, I. Feussner, Linoleic acid positioning in psi factor producing oxygenase $\mathrm{A}$, a fusion protein with an atypical cytochrome P450 activity, FEBS J. 279 (2012) 1594-1606.

[22] A.-L. Tsai, R.J. Kulmacz, Prostaglandin H synthase: resolved and unresolved mechanistic issues, Arch. Biochem. Biophys. 493 (2010) 103-124.

[23] M.W. Roessle, R. Klaering, U. Ristau, B. Robrahn, D. Jahn, T. Gehrmann, P. Konarev, A. Round, S. Fiedler, C. Hermes, D. Svergun, Upgrade of the small-angle X-ray scattering beamline X33 at the European Molecular Biology Laboratory, Hamburg, J. Appl. Crystallogr. 40 (2007) S190-S194.

[24] A.R. Round, D. Franke, S. Moritz, R. Huchler, M. Fritsche, D. Malthan, R. Klaering, D.I. Svergun, M. Roessle, Automated sample-changing robot for solution scattering experiments at the EMBL Hamburg SAXS station X33, J. Appl. Crystallogr. 41 (2008) 913-917.

[25] P.V. Konarev, V.V. Volkov, A.V. Sokolova, M.H.J. Koch, D.I. Svergun, PRIMUS: a Windows PC-based system for small-angle scattering data analysis, J. Appl. Crystallogr. 36 (2003) 1277-1282.

[26] A. Guinier, La diffraction des rayons X aux tres petits angles; application a l'etude de phenomenes ultramicroscopiques, Ann. Phys. (Paris) 12 (1939) 161-237.

[27] D.I. Svergun, Determination of the regularization parameter in indirect-transform methods using perceptual criteria, J. Appl. Crystallogr. 25 (1992) 495-503.

[28] G. Porod, General Theory, Academic Press London, 1982.

[29] D. Franke, D.I. Svergun, DAMMIF, a program for rapid ab-initio shape determination in small-angle scattering, J. Appl. Crystallogr. 42 (2009) 342-346.

[30] V.V. Volkov, D.I. Svergun, Uniqueness of ab initio shape determination in small-angle scattering, J. Appl. Crystallogr. 36 (2003) 860-864

[31] M.V. Petoukhov, D. Franke, A.V. Shkumatov, G. Tria, A.G. Kikhney, M. Gajda, C. Gorba, H.D.T. Mertens, P.V. Konarev, D.I. Svergun, New developments in the ATSAS program package for small-angle scattering data analysis, J. Appl. Crystallogr. 45 (2012) 342-350.

[32] M.B. Kozin, D.I. Svergun, Automated matching of high- and low-resolution structural models, J. Appl. Crystallogr. 34 (2001) 33-41.

[33] M.V. Petoukhov, D.I. Svergun, Global rigid body modeling of macromolecular complexes against small-angle scattering data, Biophys. J. 89 (2005) 1237-1250.

[34] A. Shevchenko, M. Wilm, O. Vorm, M. Mann, Mass spectrometric sequencing of proteins silver-stained polyacrylamide gels, Anal. Chem. 68 (1996) 850-858.

[35] M. Pannier, S. Veit, A. Godt, G. Jeschke, H.W. Spiess, Dead-time free measurement of dipole-dipole interactions between electron spins, J. Magn. Reson. 142 (2000) 331-340.

[36] G. Jeschke, V. Chechik, P. Ionita, A. Godt, H. Zimmermann, J. Banham, C.R. Timmel, D. Hilger, H. Jung, DeerAnalysis2006-a comprehensive software package for analyzing pulsed ELDOR data, Appl. Magn. Reson. 30 (2006) 473-498.

[37] F. Jerneren, A. Sesma, M. Francheschetti, M. Hamberg, E.H. Oliw, Gene deletion of 7,8-linoleate diol synthase of the rice blast fungus, J. Biol. Chem. 285 (2010) 5308-5316.

[38] U. Garscha, E.H. Oliw, Steric analysis of 8-hydroxy- and 10-hydroxyoctadecadienoic acids and dihydroxyoctadecadienoic acids formed from 8R-hydroperoxyoctadecadienoic acid by hydroperoxide isomerases, Anal. Biochem. 367 (2007) 238-246.

[39] E.H. Oliw, C. Su, T. Skogstrom, G. Benthin, Analysis of novel hydroperoxides and other metabolites of oleic, linoleic, and linolenic acids by liquid chromatography-mass spectrometry with ion trap MSn, Lipids 33 (1998) 843-852.

[40] E.H. Oliw, U. Garscha, T. Nilsson, M. Cristea, Payne rearrangement during analysis of epoxyalcohols of linoleic and alpha-linolenic acids by normal phase liquid chromatography with tandem mass spectrometry, Anal. Biochem. 354 (2006) 111-126.

[41] E.H. Oliw, A. Wennman, I. Hoffmann, U. Garscha, M. Hamberg, F. Jernerén, Stereoselective oxidation of regioisomeric octadecenoic acids by fatty acid dioxygenases, J. Lipid Res. 52 (2011) 1995-2004.

[42] D. Sirim, M. Widmann, F. Wagner, J. Pleiss, Prediction and analysis of the modular structure of cytochrome P450 monooxygenases, BMC Struct. Biol. 10 (2010) 34.

[43] H.Y. Li, T.L. Poulos, The structure of the cytochrome p450BM-3 haem domain complexed with the fatty acid substrate, palmitoleic acid, Nat. Struct. Biol. 4 (1997) 140-146.

[44] T.C. Pochapsky, S. Kazanis, M. Dang, Conformational plasticity and structure/function relationships in cytochromes P450, Antioxid. Redox Signal. 13 (2010) 1273-1296. 